\title{
Assessment and Validation of the Internal Gross Tumour Volume of Gastroesophageal Junction Cancer During Simultaneous Integrated Boost Radiotherapy
}

Jinming Shi

Cancer Hospital Chinese Academy of Medical Sciences

Yuan Tang

Cancer Hospital Chinese Academy of Medical Sciences

Ning Li

Cancer Hospital Chinese Academy of Medical Sciences

\section{Yongwen Song}

Cancer Hospital Chinese Academy of Medical Sciences

\section{Shulian Wang}

Cancer Hospital Chinese Academy of Medical Sciences

\section{Yueping Liu}

Cancer Hospital Chinese Academy of Medical Sciences

Hui Fang

Cancer Hospital Chinese Academy of Medical Sciences

Ningning Lu

Cancer Hospital Chinese Academy of Medical Sciences

\section{Yu Tang}

Cancer Hospital Chinese Academy of Medical Sciences

Shunan Qi

Cancer Hospital Chinese Academy of Medical Sciences

\section{Bo Chen}

Cancer Hospital Chinese Academy of Medical Sciences

Yexiong Li

Cancer Hospital Chinese Academy of Medical Sciences

Wenyang Liu

Cancer Hospital Chinese Academy of Medical Sciences Jing Jin ( $\nabla_{\text {jinjing@csco.org.cn ) }}$

Cancer Hospital Chinese Academy of Medical Sciences 
Research

Keywords: gastroesophageal junction (GEJ) cancer, neoadjuvant, radiotherapy, internal gross tumour volume, simultaneous integrated boost radiotherapy

Posted Date: June 14th, 2021

DOl: https://doi.org/10.21203/rs.3.rs-576513/v1

License: (c) (i) This work is licensed under a Creative Commons Attribution 4.0 International License.

Read Full License

Version of Record: A version of this preprint was published at Radiation Oncology on February 3rd, 2022. See the published version at https://doi.org/10.1186/s13014-022-01996-6. 


\section{Abstract}

\section{Background}

Respiratory motion may introduce errors during radiotherapy. This study aims to assess and validate internal gross tumour volume (IGTV) margins in different regions in gastroesophageal junction (GEJ) tumours during simultaneous integrated boost radiotherapy.

\section{Methods}

We enrolled 10 patients in group A and 9 patients in group B. For all patients, two markers were placed at the upper and lower boundaries of tumour before treatment. In group A, within the simulation and every five fractions of radiotherapy, we used 4-dimensional computed tomography (4DCT) to record the intrafractional displacement of the proximal and distal markers. By fusing the average image of each fraction with the simulation image according to the lumbar vertebra, the interfractional displacement could be obtained. We calculated the IGTV margin in the proximal and distal regions of the GEJ tumour. In group $B$, by referring to the simulation images and cone-beam computed tomography (CBCT) images, the range of tumour displacement in different regions within the GEJ tumour was estimated. We verified the proportion of tumours with marker displacement within the range obtained in group $A$ to estimate the accuracy of the IGTV margin.

Results

The intrafractional displacement in the cranio-caudal (CC) direction was significantly larger than that in the anterior-posterior (AP) and left-right (LR) directions for both the proximal and distal markers of the tumour. The interfractional displacement in the AP and LR directions was larger than that in the CC direction ( $p=0.001, p=0.017)$ according to the distal marker. The IGTV margins for in the LR, AP and CC directions were $9 \mathrm{~mm}, 8.5 \mathrm{~mm}$ and $12.1 \mathrm{~mm}$ for proximal marker and $15.8 \mathrm{~mm}, 12.7 \mathrm{~mm}$ and $11.5 \mathrm{~mm}$

for distal marker. In group B, the proportions of tumours that fell within the IGTV margin in the LR, AP and CC directions were $96.5 \%, 91.3 \%$ and $96.5 \%$ for the proximal marker and $100 \%, 96.5 \%, 93.1 \%$ for the distal marker.

\section{Conclusions}

Our study proposed individualized IGTV margins for different regions within GEJ tumours during neoadjuvant radiotherapy. The IGTV margin determined in this study was relatively small and was validated appropriately. It could be a reference in clinical practice.

\section{Introduction}

Gastric cancer ranks fifth in morbidity and third in mortality worldwide, and the percentage of gastroesophageal junction (GEJ) cancer has increased rapidly in recent years [1]. For locally advanced GEJ cancer, Shapiro confirmed the survival advantages of neoadjuvant chemoradiotherapy (CRT) over 
surgery alone [2]. Stahl showed that neoadjuvant CRT can result in significant downstaging and a greater local control benefit than neoadjuvant chemotherapy [3]. Neoadjuvant CRT is a standard treatment widely used in locally advanced GEJ cancer. In recent years, there has been a trend of applying simultaneous integrated boost (SIB) radiotherapy in GEJ and oesophageal cancer [4]. Therefore, precise definition of the target volume is crucial for radiotherapy.

The GEJ connects the stomach and oesophagus, so the treatment process will be affected by respiration, gastric filling status, gastric peristalsis factors and so on $[5,6]$. Interfractional and intrafractional tumour displacements result in inaccuracies during treatment. According to the International Commission on Radiation Units and Measurements (ICRU) 62 report, the planning target should not only include the clinical target volume but also cover the tumour displacement margin and setup errors [7]. Therefore, it is essential to understand how GEJ cancer moves during radiotherapy.

Defining a tight internal gross tumour volume (IGTV) margin may improve tumour targeting and avoid unnecessary exposure to normal tissue. In oesophageal cancer and liver cancer, the internal tumour margin varies according to the tumour location [8, 9]. Similarly, in GEJ cancer, the IGTV margin should also be based on the tumour location. However, a consensus on the IGTV margin has not yet been established. To date, several studies have explored the primary tumour motion of gastric cancer or GEJ cancer, but none of them verified the accuracy of their margin $[10,11]$. The present study assessed and validated the IGTV margin in proximal and distal GEJ cancer with fiducial markers.

\section{Materials And Methods}

Nineteen patients pathologically diagnosed with adenocarcinoma of the GEJ were enrolled in this study. All patients underwent neoadjuvant chemoradiotherapy at a dose of $45 \mathrm{~Gy}$ in 25 fractions. The study design is presented in Fig. 1. Groups A and B contained ten patients and nine patients, respectively, to assess and validate the IGTV margin.

Group A: assess the IGTV margin

From October 2018 to June 2019, we prospectively enrolled ten patients with locally advanced GEJ cancer who received neoadjuvant chemoradiotherapy. All patients were selected from a single pilot study (clinical trial: NCT04062058). Before the simulation, a gastroenterologist placed two markers in the submucosal layers at the cranial and caudal border of the tumour. The proximal marker was located at the GEJ, and the distal marker was located at the lesser gastric curvature.

During the simulation, to maintain a consistent stomach volume, the patients were instructed to fast for at least 4 hours and drink $300 \mathrm{ml}$ semifluid ten minutes before the planning four-dimensional computed tomography (4DCT) simulation. Patients laid on the bed in the supine position with their arms crossed above their heads. All patients were stabilized by a thermoplastic mask under free-breathing conditions. Every five fractions during treatment, patients were required to repeat this process with 4DCT technology in the simulation room. These 4DCT images were substituted for the images during the real therapy. Each 
4DCT image was reconstructed into 10 phases of a free-breathing cycle from $0-90 \%$, and an average CT image was derived automatically. All 4DCT images were transferred to the Pinnacle ${ }^{3} 9.1$ treatment planning system (Pinnacle ${ }^{3}$, version 9.1, Philips Medical Systems, Eindhoven, The Netherlands). Overall, each patient had five sets of 4DCT images and four sets of 4DCT images for intrafractional and interfractional motion analysis, respectively. Initially, we obtained 50 sets of 4DCT images during the simulation and radiotherapy; however, two patients dropped their markers at the beginning of radiotherapy, and we lost 8 sets of 4DCT images during radiotherapy. Hence, there were 32 sets of images to analyse the interfractional tumour displacement and 42 sets of images to analyse the intrafractional tumour displacement.

Next, we fused the $0 \%-90 \%$ respiration images with the average image according to the lumbar vertebra and delineated the outline of the markers on the image of each phase under the bone window. After contouring the outline of the marker, the geometric centre coordinates were determined automatically by the planning system. By subtracting the average image's three-dimensional coordinates in different respiration phases, we could determine the intrafractional tumour displacement in the left-right (LR), anterior-posterior (AP), and cranio-caudal (CC) directions.

In regard to interfractional tumour displacement, we fused the average images obtained during radiotherapy with the simulation images. By subtracting their coordinates, we obtained the interfractional displacement. In the end, to ensure a minimum dose of $95 \%$ of the target volume in $90 \%$ of patients, the internal gross target volume (IGTV) in different directions should be $2.5 \Sigma+0.7 \sigma$, according to the formula proposed by Van Herk[12]. We calculated the systematic error $(\Sigma)$ and random error $(\sigma)$ of inter-/intrafractional displacement of the tumour and consequently obtained the IGTV margin.

Group B: validate the IGTV margin

For further investigations, we enrolled 9 patients in group B to validate the accuracy of the IGTV margin determined in group A. Patients who met the following inclusion criteria were included: (1) diagnosed with GEJ cancer and received neoadjuvant radiotherapy between January 2010 and July 2020; (2) radiotherapy performed under free-breathing conditions. (3) at least two markers implanted at the cranial and caudal border of the tumour; and (4) five to nine CBCT images were acquired during therapy. In the early stages of radiotherapy, two markers were lost the proximal and distal regions of the tumour. Finally, 14 markers were visible in the proximal and distal regions of the tumour during therapy, and 58 sets of $\mathrm{CBCT}$ images were included in the analysis.

To reduce setup errors, we fused the patients' $\mathrm{CBCT}$ images with their simulation images and sent all images to the Pinnacle ${ }^{3} 9.1$ treatment planning system. Similarly, by delineating the outline of the marker and subtracting the marker coordinates during therapy from the simulation images, we could obtain the interfractional tumour displacement. Finally, we validated the proportion of tumours with of marker displacement within the IGTV margin determined in group A.

Statistical Analysis

Page 5/15 
All statistical analyses were performed by using SPSS version 22.0. The results are presented as the mean \pm standard deviation. A pairwise t-test was used to compare the deviations between directions. A Pvalue $<0.05$ was considered statistically significant.

\section{Results}

In group A, all patients were diagnosed with locally advanced GEJ cancer considered clinical stage T3-4 or $\mathrm{N}$ positive. Two patients $(20 \%)$ had Siewert's type $\otimes$ tumours, and eight patients $(80 \%)$ had Siewert's

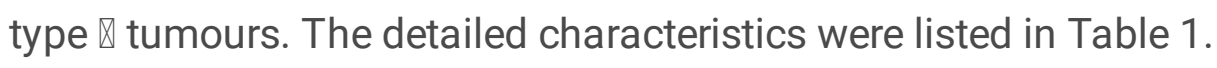

Table 1 characteristics of patients and tumour in two groups 


\begin{tabular}{|c|c|c|}
\hline characteristics & $\begin{array}{l}\text { Group A } \\
{[n=10]}\end{array}$ & $\begin{array}{l}\text { Group B } \\
{[n=9]}\end{array}$ \\
\hline \multicolumn{3}{|l|}{ Age } \\
\hline median (range) & $64(54-64)$ & $56(36-69)$ \\
\hline \multicolumn{3}{|l|}{ Gender (n, \%) } \\
\hline Male & $9(90)$ & $6(66.7)$ \\
\hline Female & $1(10)$ & $3(33.3)$ \\
\hline \multicolumn{3}{|l|}{ Siewert type (n, \%) } \\
\hline Siewert $\otimes$ & $0(0)$ & $0(0)$ \\
\hline Siewert $\otimes$ & $2(20)$ & $4(44.4)$ \\
\hline Siewert $\nabla$ & $8(80)$ & $5(55.6)$ \\
\hline \multicolumn{3}{|c|}{ Tumour upper boundary to incisor } \\
\hline median(cm, range) & $39.0(35-43)$ & $40(35-49)$ \\
\hline \multicolumn{3}{|c|}{ Tumour lower boundary to incisor } \\
\hline median( $\mathrm{cm}$, range) & $45.5(40-50)$ & $46(42-53)$ \\
\hline \multicolumn{3}{|l|}{ Tumour length } \\
\hline median(cm, range) & $6(4-8)$ & $6(4-13)$ \\
\hline \multicolumn{3}{|c|}{ Clinical T stage $(n, \%)$} \\
\hline T3 & $1(10)$ & $1(11.1)$ \\
\hline $\mathrm{T} 4 \mathrm{a}$ & $9(90)$ & $7(77.8)$ \\
\hline $\mathrm{T} 4 \mathrm{~b}$ & 0 & $1(11.1)$ \\
\hline \multicolumn{3}{|c|}{ Clinical N stage $(n, \%)$} \\
\hline No & $1(10)$ & 0 \\
\hline N1 & $5(50)$ & 0 \\
\hline N2 & $3(30)$ & $7(77.8)$ \\
\hline N3 & $1(10)$ & $2(22.2)$ \\
\hline \multicolumn{3}{|c|}{ Clinical TNM stage (n, \%) } \\
\hline ૧ & $1(10)$ & 0 \\
\hline$\llbracket \mathrm{A}$ & $5(50)$ & 0 \\
\hline
\end{tabular}




\begin{tabular}{|lll|}
\hline characteristics & $\begin{array}{l}\text { Group A } \\
{[\mathbf{n = 1 0 ]}}\end{array}$ & $\begin{array}{l}\text { Group B } \\
{[\mathbf{n}=9]}\end{array}$ \\
\hline$\triangle B$ & $3(30)$ & $7(77.8)$ \\
\hline$ه C$ & $1(10)$ & $2(22.2)$ \\
\hline
\end{tabular}

The intrafractional and interfractional tumour displacements of the proximal markers and distal markers for GEJ cancer are listed in Table 2. The deviations between directions are shown in Fig. 2. In one respiration phase, the intrafractional displacement in the $\mathrm{CC}$ direction was significantly larger than that in other directions for both the proximal and distal markers of the tumour. When comparing the interfractional tumour displacement among different directions, the proximal marker showed the largest displacement in the CC direction followed by in the AP direction $(p=0.003)$. For the distal marker, the interfractional tumour displacements in the LR and AP directions was larger than that in the CC direction $(p=0.001, p=0.017)$.

For intrafractional displacement, no significant difference was seen between the proximal or distal markers. Regarding interfractional displacement, in the CC direction, the proximal and distal markers did not differ significantly $(p=0.933)$, but in the LR and AP directions, the displacement of the distal marker was larger than that of the proximal markers.

Table 2 The intrafractional and interfractional tumour displacements in Group A \& B (mean \pm standard deviation, range)

\begin{tabular}{|c|c|c|c|c|}
\hline & Displacement & Direction & Proximal (mm) & Distal(mm) \\
\hline \multirow[t]{6}{*}{ Group A } & \multirow[t]{3}{*}{ Intrafraction } & LR & $2.25 \pm 1.29(0.3 \sim 5.5)$ & $2.38 \pm 1.8(0.1 \sim 9.2)$ \\
\hline & & AP & $3.64 \pm 1.82(0.5 \sim 11.2)$ & $3.33 \pm 1.69(0.3 \sim 6.4)$ \\
\hline & & $\mathrm{CC}$ & $7.78 \pm 3.81(0.5 \sim 21.5)$ & $7.19 \pm 2.84(1.2 \sim 16.1)$ \\
\hline & \multirow[t]{3}{*}{ Interfraction } & LR & $3.50 \pm 3.85(0 \sim 15.6)$ & $8.13 \pm 6.7(0.3 \sim 28.5)$ \\
\hline & & $\mathrm{AP}$ & $2.31 \pm 2.73(0 \sim 13.0)$ & $6.33 \pm 4.73(0.3 \sim 22.1)$ \\
\hline & & $\mathrm{CC}$ & $4.38 \pm 4.28(0 \sim 16.3)$ & $4.13 \pm 3.68(0 \sim 15.6)$ \\
\hline \multirow[t]{3}{*}{ Group B } & Interfraction & LR & $3.70 \pm 2.87(0.1 \sim 11.2)$ & $5.39 \pm 3.76(0 \sim 14.8)$ \\
\hline & & $\mathrm{AP}$ & $3.32 \pm 3.12(0.1 \sim 13.4)$ & $4.43 \pm 4.06(0.2 \sim 20.2)$ \\
\hline & & $\mathrm{CC}$ & $4.79 \pm 3.36(0 \sim 14.1)$ & $6.1 \pm 3.96(0.4 \sim 14.5)$ \\
\hline
\end{tabular}


Table 3 shows the systematic error $(\Sigma)$ and the random error $(\sigma)$ of the proximal and distal markers based on the interfractional and intrafractional displacements. Finally, for the tumour marker proximal to the chest, the IGTV margins in the LR, AP and CC directions were $9 \mathrm{~mm}, 8.5 \mathrm{~mm}$ and $12.1 \mathrm{~mm}$, respectively. For the tumour marker distal to the chest, the IGTV margins in the LR, AP and CC directions were 15.8 $\mathrm{mm}, 12.7 \mathrm{~mm}$ and $11.5 \mathrm{~mm}$, respectively.

Table 3 The IGTV margin in different directions

\begin{tabular}{|c|c|c|c|c|c|c|c|c|c|}
\hline & \multicolumn{3}{|c|}{$\mathrm{LR}(\mathrm{mm})$} & \multicolumn{3}{|c|}{$\mathrm{AP}(\mathrm{mm})$} & \multicolumn{3}{|c|}{$\mathrm{cc}(\mathrm{mm})$} \\
\hline & $\Sigma$ & $\sigma$ & range & $\Sigma$ & $\sigma$ & range & $\Sigma$ & $\sigma$ & range \\
\hline Proximal & 2.96 & 2.28 & 9 & 2.45 & 3.37 & 8.5 & 3.19 & 5.87 & 12.1 \\
\hline distal & 4.78 & 5.40 & 15.8 & 4.05 & 3.64 & 12.7 & 3.60 & 3.50 & 11.5 \\
\hline
\end{tabular}

In group $B$, four patients had Siewert's type $\otimes$ tumours, and five patients had Siewert's type $\mathbb{Q}$ tumours (refer to Table 1). Table 2 shows the interfractional tumour displacement in group B. Based on the IGTV margins calculated in group A, Fig. 3 shows that the proportion of tumours within the IGTV margin in the LR, AP and CC directions were $96.5 \%, 91.3 \%$ and $96.5 \%$ for the proximal marker and $100 \%, 96.5 \%, 93.1 \%$ for the distal marker, respectively.

\section{Discussion}

During neoadjuvant radiotherapy, gross tumours in the GEJ may move significantly due to many reasons. Although the movement of GEJ tumours may influence the accuracy of the dose delivery to the GTV, few studies have focused on the IGTV margin for GEJ cancer. In this study, GEJ cancer was divided into different regions, and the IGTV margin was assessed by 4DCT technology. In addition, we enrolled a separate group to validate the accuracy of the IGTV margin. Our studies offer a reference for IGTV margin expansion, especially during SIB intensity-modulated radiotherapy (IMRT) or for institutions lacking image-guided radiotherapy technology.

We delineated the outline of markers under the bone window, which may reduce the uncertainties of contouring the GTV. Moreover, compared to traditional CT simulations, 4DCT technology records temporal and spatial organ motion[13], improving the reliability of the data in our study. When discussing intrafractional motion, Peng Jin et al. enrolled twenty oesophageal cancer patients, and sixty markers were implanted in the proximal, middle, distal oesophagus and proximal stomach of these patients, including seventeen markers located in the proximal stomach. The study found that the intrafractional motion of the proximal stomach in LR, AP and CC directions was $3.7 \mathrm{~cm}, 5.3 \mathrm{~cm}$, and $8.2 \mathrm{~cm}$, respectively. They also discovered that the closer the oesophageal cancer is to the mediastinum, the larger the tumour 
movement in all directions[14] which is similar to ours. In addition, Lever et al. calculated oesophageal cancer motion by cine-magnetic resonance imaging and found that the tumour motion in the CC direction was larger than that in the AP and LR directions[15], which is consistent with our study. In our study, regardless of the marker position, the intrafractional displacement in the CC direction was always moves greatest, which is largely attributed to respiration[16].

In regard to interfractional motion, wang et al. discovered that the difference in interfractional motion in different directions was not significant for thoracic oesophageal cancer[17]. However, wang et al. found that the interfractional motion of oesophageal cancer was larger in the CC direction than other directions[18], which is similar to the data from the proximal marker in our study. However, the distal marker showed larger interfractional motion in the AP and LR directions in our study. On the one hand, it is likely that changes in stomach volume and shape during each fraction may be more affected in the AP and LR directions when the tumour is closer to the stomach. Studies have proven that respiratory gating[19], abdominal compression[20], and active breath control[21] technologies may reduce the errors caused by respiration; however, these methods are complicated and time consuming and require specific technology and cooperation from patients.

We calculated the IGTV margins in the proximal and distal regions within GEJ tumours. For tumour centres closer to the GEJ, we can refer to the proximal IGTV margin, and for tumours near the lesser curvature of the stomach, the distal IGTV margin can be referenced. Furthermore, we verified that the IGTV margin was appropriate in nine patients. Peng Jin et al. enrolled eleven patients diagnosed with proximal gastric cancer, and the recommended IGTVs in the LR, AP and CC directions were $16.4 \mathrm{~mm}, 6.4$ $\mathrm{mm}$, and $14.6 \mathrm{~mm}$, respectively[22]; however, their data were merely calculated based on interfractional motion and did not account for gastric fasting or filling. Watanabe et al. analysed six gastric lymphoma patients by using repeated CT scans and suggested that to avoid missing the radiotherapy target, the expansion margins from the whole stomach in the LR, AP and CC directions should be $31 \mathrm{~mm}, 29.6 \mathrm{~mm}$, and $15.9 \mathrm{~mm}$, respectively[10]. Similar to our study, the closer the tumour is to the gastric body, the larger the IGTV margin in the LR and AP directions. The European Organisation for Research and Treatment of Cancer (EORTC) guidelines recommended that the IGTV margin of GEJ cancer in LR, AP and superior direction should be $10 \mathrm{~mm}$ and that in the inferior direction should be $15 \mathrm{~mm}$. For gastric cancer, the recommended margin in three dimensions was $15 \mathrm{~mm}[23]$. The expansion margin in our study was based on advanced 4DCT technology, which is more accurate in these directions. The application of these margins is expected to reduce side effects during radiotherapy.

GEJ cancer lacks IGTV recommendations during neoadjuvant radiotherapy. Our study not only assessed the IGTV margin in different regions within GEJ tumours but also verified its accuracy for the first time. The IGTV margin is vital when using SIB IMRT to treat GEJ cancer. However, this study had some limitations that should be emphasize. First, our sample size was limited in groups A and B. Second, the $4 \mathrm{DCT}$ and $\mathrm{CBCT}$ images represent only a short time of tumour displacement and cannot represent the real-time displacement during radiotherapy. Future work may focus on prospective clinical trials and measure radiotherapy target accuracy and toxicity based on the IGTV margin determined in this study. 


\section{Conclusions}

In conclusion, during neoadjuvant radiotherapy for GEJ cancer, the IGTV margin in our study was relatively small and feasible and may serve as a reference in clinical practice.

\section{Abbreviations}

AP: anterior-posterior, $\mathrm{CT}$ : computed tomography, CBCT: cone-beam computed tomography, CC: craniocaudal, CRT: chemoradiotherapy, EORTC: European Organisation for Research and Treatment of Cancer, GTV: gross tumour volume, GEJ: gastroesophageal junction, IGTV: internal gross tumour volume, ICRU: International Commission on Radiation Units and Measurements, IMRT: Intensity-modulated radiotherapy, LR: left-right, SIB: simultaneous integrated boost, 4DCT: 4-dimensional computed tomography

\section{Declarations}

\section{Ethics approval and consent to participate}

This study met the rapid review conditions and institutional review board approval was obtained for this trial from the ethical committee of the Chinese Academy of Medical Sciences $\mathbb{N C C} 1829 \rrbracket$.

\section{Consent for publication}

Not applicable

\section{Availability of data and materials}

The datasets used and analysed during the current study are available from the corresponding author on reasonable request.

\section{Competing interests}

The authors declare that they have no competing interests.

\section{Funding}

This study was supported by the Grants from Natural Science Foundation of China (No. 82073352) and Capital's Funds for Health Improvement and Research (2020-1-4021).

\section{Author's contributions}

JMS, WYL and JJ made great contribution to design this study. JMS drafted this manuscript, WYL and JJ revised the manuscript carefully. YT and NL contributed greatly into the treatment of patients; YWS, 
SLW, YPL, HF, NNL, YT, SNQ, BC and YXL participated in enrolling patients to this group and conducting the study. All authors read and approved the final manuscript.

\section{Acknowledgements}

We thank all patients and clinicians participated in this study sincerely.

\section{References}

1. Bray F, Ferlay J, Soerjomataram I, et al. Global cancer statistics 2018: GLOBOCAN estimates of incidence and mortality worldwide for 36 cancers in 185 countries [J]. CA Cancer J Clin, 2018, 68(6): 394-424. doi:10.3322/caac.21492

2. Shapiro J, Van Lanschot J J B, Hulshof M C C M, et al. Neoadjuvant chemoradiotherapy plus surgery versus surgery alone for oesophageal or junctional cancer (CROSS): long-term results of a randomised controlled trial [J]. The Lancet Oncology, 2015, 16(9): 1090-1098. doi:10.1016/s14702045(15)00040-6

3. Stahl M, Walz M K, Riera-Knorrenschild J, et al. Preoperative chemotherapy versus chemoradiotherapy in locally advanced adenocarcinomas of the oesophagogastric junction (POET): Long-term results of a controlled randomised trial [J]. Eur J Cancer, 2017, (81)183-190. doi:10.1016/j.ejca.2017.04.027

4. Chen $\mathrm{D}$, Menon $\mathrm{H}$, Verma $\mathrm{V}$, et al. Results of a Phase $1 / 2$ Trial of Chemoradiotherapy With Simultaneous Integrated Boost of Radiotherapy Dose in Unresectable Locally Advanced Esophageal Cancer [J]. JAMA Oncol, 2019, 5(11): 1597-1604. doi:10.1001/jamaoncol.2019.2809

5. Van De Voorde L, Larue R, Persoon L, et al. The influence of gastric filling instructions on dose delivery in patients with oesophageal cancer: A prospective study [J]. Radiother Oncol, 2015, 117(3): 442-447. doi:10.1016/j.radonc.2015.09.006

6. Kincaid R E, Jr., Hertanto A E, Hu Y C, et al. Evaluation of respiratory motion-corrected cone-beam CT at end expiration in abdominal radiotherapy sites: a prospective study [J]. Acta Oncol, 2018, 57(8): 1017-1024. doi:10.1080/0284186x.2018.1427885

7. Stroom J C, Heijmen B J. Geometrical uncertainties, radiotherapy planning margins, and the ICRU-62 report [J]. Radiother Oncol, 2002, 64(1): 75-83. doi:10.1016/s0167-8140(02)00140-8

8. Tsai Y L, Wu C J, Shaw S, et al. Quantitative analysis of respiration-induced motion of each liver segment with helical computed tomography and 4-dimensional computed tomography [J]. Radiat Oncol, 2018, 13(1): 59. doi:10.1186/s13014-018-1007-0

9. Voncken F E M, Nakhaee S, Stam B, et al. Quantification of Esophageal Tumor Motion and Investigation of Different Image-Guided Correction Strategies [J]. Pract Radiat Oncol, 2020, 10(2): 8492. doi:10.1016/j.prro.2019.11.012

10. Watanabe $M$, Isobe $K$, Uno $T$, et al. Intrafractional gastric motion and interfractional stomach deformity using CT images [J]. J Radiat Res, 2011, 52(5): 660-665. doi:10.1269/jrr.11018 
11. Machiels $M$, Jin $P$, Van Hooft $J$ E, et al. Reduced inter-observer and intra-observer delineation variation in esophageal cancer radiotherapy by use of fiducial markers [J]. Acta Oncol, 2019, 58(6): 943-950. doi:10.1080/0284186X.2019.1588991

12. Van Herk M. Errors and margins in radiotherapy [J]. Semin Radiat Oncol, 2004, 14(1): 52-64. DOI:10.1053/j.semradonc.2003.10.003

13. Lischalk J W, Kole T P, Anjum H M, et al. Four-dimensional computed tomography prediction of interand intrafractional upper gastrointestinal tumor motion during fractionated stereotactic body radiation therapy [J]. Pract Radiat Oncol, 2016, 6(3): 176-182. doi:10.1016/j.prro.2015.10.006

14. Jin P, Hulshof M C, De Jong R, et al. Quantification of respiration-induced esophageal tumor motion using fiducial markers and four-dimensional computed tomography [J]. Radiother Oncol, 2016, 118(3): 492-497. doi:10.1016/j.radonc.2016.01.005

15. Lever F M, Lips I M, Crijns S P, et al. Quantification of esophageal tumor motion on cine-magnetic resonance imaging [J]. International journal of radiation oncology, biology, physics, 2014, 88(2): 419424. doi:10.1016/j.ijrobp.2013.10.036

16. Uchinami Y, Suzuki R, Katoh N, et al. Impact of organ motion on volumetric and dosimetric parameters in stomach lymphomas treated with intensity-modulated radiotherapy [J]. Journal of applied clinical medical physics, 2019, 20(8): 78-86. doi:10.1002/acm2.12681

17. Wang J Z, Li J B, Wang W, et al. Changes in tumour volume and motion during radiotherapy for thoracic oesophageal cancer [J]. Radiother Oncol, 2015, 114(2): 201-205. doi:10.1016/j.radonc.2014.12.010

18. Wang J, Lin S H, Dong L, et al. Quantifying the interfractional displacement of the gastroesophageal junction during radiation therapy for esophageal cancer [J]. International journal of radiation oncology, biology, physics, 2012, 83(2): e273-280. doi:10.1016/j.jijrobp.2011.12.048

19. Kim J I, Lee H, Wu H G, et al. Development of patient-controlled respiratory gating system based on visual guidance for magnetic-resonance image-guided radiation therapy [J]. Med Phys, 2017, 44(9): 4838-4846. doi:10.1002/mp.12447

20. Raphael J C, Varghese M K, Gopu P G, et al. Effectiveness of abdominal compression in reducing internal target motion during conformal radiotherapy for carcinoma stomach - A pilot study [J]. Indian journal of cancer, 2018, 55(3): 226-229. doi:10.4103/ijc.IJC_95_18

21. Lu L, Diaconu C, Djemil T, et al. Intra- and inter-fractional liver and lung tumor motions treated with SBRT under active breathing control [J]. Journal of applied clinical medical physics, 2018, 19(1): 3945. doi:10.1002/acm2.12220

22. Jin P, Van Der Horst A, De Jong R, et al. Marker-based quantification of interfractional tumor position variation and the use of markers for setup verification in radiation therapy for esophageal cancer [J]. Radiother Oncol, 2015, 117(3): 412-418. doi:10.1016/j.radonc.2015.10.005

23. Matzinger O, Gerber E, Bernstein Z, et al. EORTC-ROG expert opinion: radiotherapy volume and treatment guidelines for neoadjuvant radiation of adenocarcinomas of the gastroesophageal 
junction and the stomach [J]. Radiother Oncol, 2009, 92(2): 164-175.

doi:10.1016/j.radonc.2009.03.018

Figures

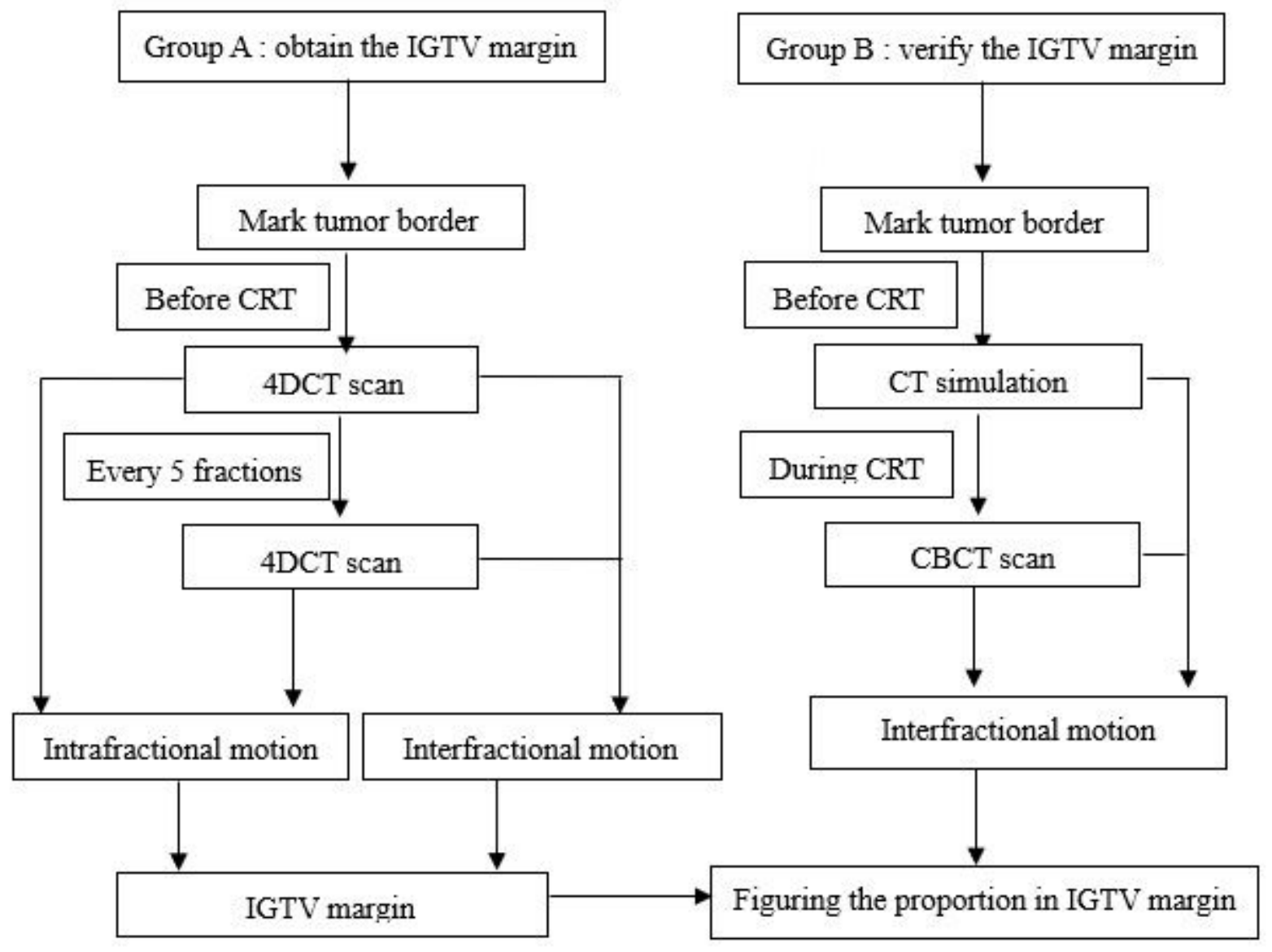

Figure 1

The flowchart of study design 


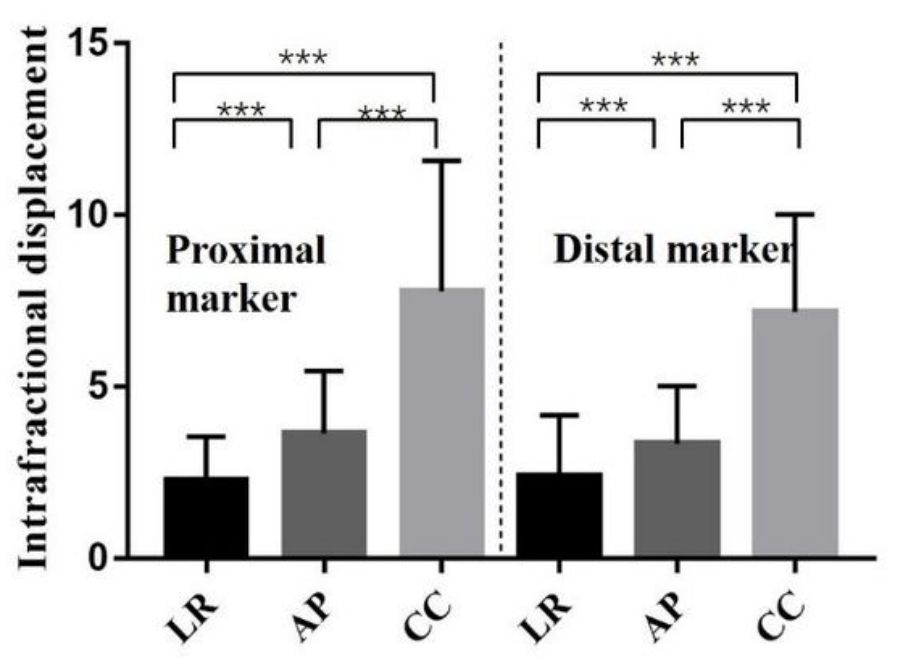

Direction

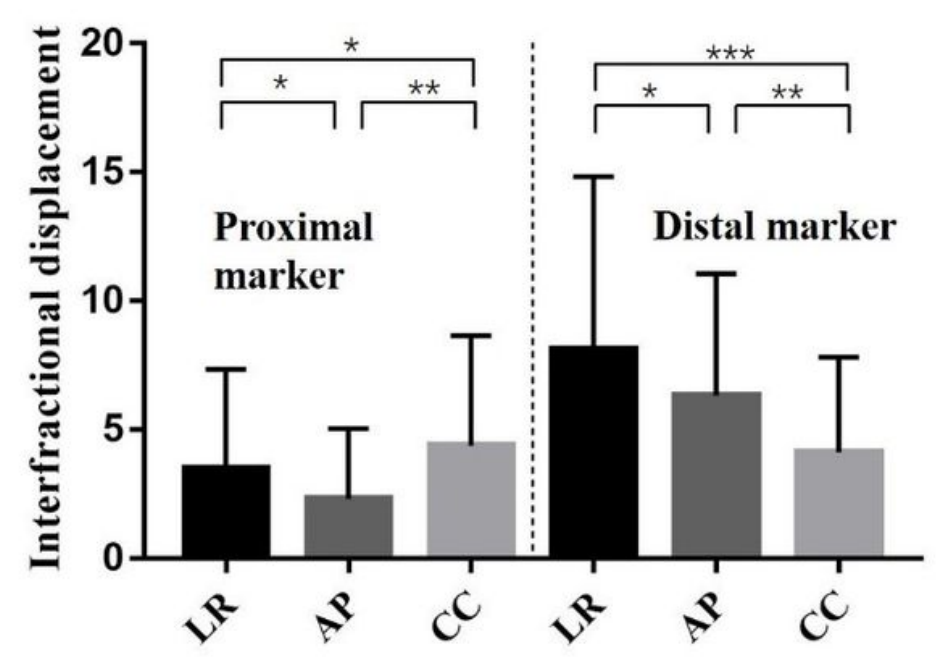

Direction

Figure 2

The deviations between different directions in proximal and distal marker $(*: p>0.05, * \star: p<0.05, * \star *$ : $\mathrm{p}<0.001)$

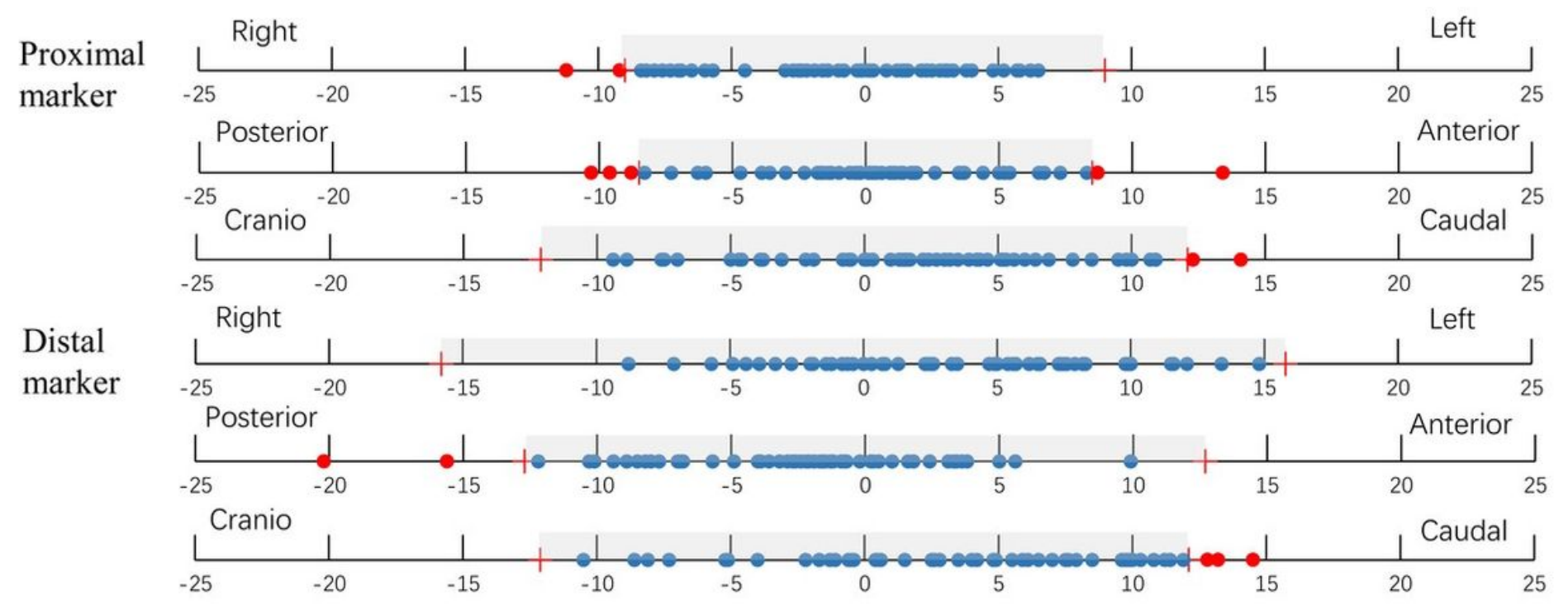

Figure 3

The distribution of markers according to the IGTV margin (grey box: the IGTV margin in different directions, blue dot: in the IGTV margin, red dot: outside the IGTV marker) 